\title{
Sindrome de ojo blanco, como causa de ceguera
}

\author{
White eye syndrome, as a cause of blindness \\ José Gustavo Avilés-Calderón', Patricia Ximena Vargas-Chang²
}

\begin{abstract}
Resumen
El Síndrome de ojo blanco como causa de ceguera busca englobar un grupo de patologías oculares cuyo desenlace es la pérdida visual. La etiología de estas enfermedades es diversa, se trata desde enfermedades asociadas a un aumento de la presión intraocular como el glaucoma de ángulo abierto, enfermedades obstructivas vasculares de los vasos retinianos principales o problemas inflamatorios del nervio óptico.
\end{abstract}

Palabras clave: Ceguera, pérdida visual.

Abstract

The white-eye syndrome as a cause of blindness encompass a group of eye diseases whose outcome is visual loss. The etiology of these diseases is diverse, ranging from diseases associated with an increase in intraocular pressure such as openangle glaucoma, vascular occlusive diseases of the principal retinal vessels or inflammatory diseases of the optic nerve.

Keywords: Blindness, visual loss.

\section{Introducción}

El síndrome de ojo blanco se refiere a un grupo de enfermedades que sin generar congestión conjuntival pueden causar morbilidad capaz de comprometer la visión del paciente; entre ellas, glaucoma primario de ángulo abierto, desprendimiento de retina, neuritis óptica, diabetes mellitus, obstrucciones vasculares, degeneración macular relacionada con la edad, etc. Lo importante es que todas estas entidades pueden ser diagnosticadas por el médico general a través del examen de fondo de ojo y ser oportunamente derivadas al médico oftalmólogo para el precoz manejo especializado.

\section{Glaucoma Primario de Ángulo Abierto}

El Glaucoma es una neuropatía óptica progresiva con pérdida de las células ganglionares que produce daño irreversible del campo visual. El Glaucoma Primario de ángulo abierto es clásicamente conocido como el "ladrón silencioso" de la visión por cursar en forma asintomática hasta las etapas más avanzadas. En el mundo la primera causa de ceguera irreversible y su prevalencia aumenta con la edad. El aumento de la presión intraocular es el principal factor de riesgo pero no el único; aunque su nivel determina el daño del nervio óptico. Para prevenir la deficiencia visual y ceguera asociadas a glaucoma debemos realizar estrategias para una detección precoz y realizar un adecuado manejo de los casos, sobre todo aquellos con un daño muy agresivo o una velocidad de progresión muy rápida ${ }^{(1)}$. El glaucoma es una neuropatía óptica progresiva que cursa con alteraciones del disco óptico caracterizada por aumento de la excavación fisiológica como consecuencia de la muerte de las células ganglionares de la retina. Existen múltiples clasificaciones para el glaucoma siendo la principal: glaucoma de ángulo abierto y glaucoma de ángulo estrecho. Siendo el $70 \%$ de los casos glaucoma primario de ángulo abierto (GPAA). La prevalencia del Glaucoma aumenta con la edad asociado a la mayor sobrevida; su prevención requiere métodos eficaces para detección precoz y tratamiento oportuno que permitan evitar ceguera irreversible.

El riesgo de pérdida visual y ceguera se reduce con un control oftalmológico adecuado en pacientes con glaucoma. Se debe realizar un diagnóstico precoz para un tratamiento oportuno considerando que hasta el $75 \%$ desconoce su diagnóstico (estudio LALES Los Angeles Eye Study). Los principales factores de riesgo son: edad mayor de 60 años, hipertensión ocular y antecedente familiar de glaucoma. La pérdida visual progresiva ocurre en etapas tardías de la enfermedad, para lo cual es necesaria la educación de la población para acudir y tener controles periódicos, evitando con ello las alteraciones irreversibles y la ceguera. El diagnóstico de glaucoma está basado en la evaluación estructural del nervio óptico y la correlación con el daño

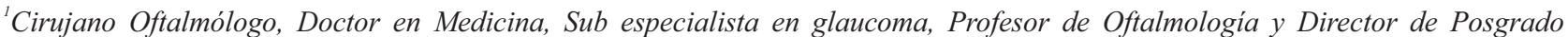
Universidad San Martín de Porres. Presidente Capítulo de Glaucoma, y Secretario Actividades Científicas Sociedad Peruana de Oftalmología. ${ }^{2}$ Cirujana Oftalmóloga, Diplomado en Glaucoma Universidad San Martín de Porres; Asistente Unidad de Glaucoma Hospital Nacional Arzobispo Loayza. 
funcional más tardío del campo visual, aún cuando la presión intraocular este dentro de límites normales ${ }^{(2)}$.

El glaucoma es la segunda causa de ceguera a nivel mundial y la primera no recuperable ${ }^{(3)}$. Para el 2020 se estima que el total de personas diagnosticadas de glaucoma en Latinoamérica (LA) podría llegar a los 8 millones (IC95\% llegaría a 13,6 millones) $)^{(4)}$, representando el $12.9 \%$ del total mundial. La prevalencia regional para LA de glaucoma en personas mayores de 40 años se calcula está en 3.6\% (IC 95\% $2.08-6.31)^{(5)}$. Se entiende que esta prevalencia de glaucoma en la población hispana es menor que en la poblaciones africanas pero mayor que la poblaciones blancas no hispanas y se espera que aumente en $\mathrm{LA}^{(6)}$ aunque la prevalencia de glaucoma varía entre los diferentes países de LA, siendo su rango entre $1 \%$ a $3.4 \%{ }^{(7,8)}$. Hasta el $75 \%$ de los pacientes con glaucoma no diagnosticados ${ }^{(9)}$ y estudios realizados en Brasil, reportan una visión mejor corregida en su primera consulta, menor o igual a $20 / 200$ en el $53.6 \%$ del peor ojo y $13.4 \%$ del mejor ojo. Una relación copa disco se encontró entre 0.8 y 1 en el $67,7 \%$ de los casos (en el peor ojo) y en el 58,8\% (del mejor ojo) ${ }^{(3)}$. Por este motivo es importante el diagnóstico precoz y el manejo oportuno de casos no complicados por el oftalmólogo en la atención primaria, refiriéndose los casos avanzados o complejos o quirúrgicos a los especialistas ${ }^{(3)}$.

Además de tomar nota de los factores de riesgo asociados anteriormente descritos, el médico general debe dominar el reconocimiento de las estructuras en el fondo de ojo: el Nervio, arquitectura vascular, retina y mácula.

Para sospechar el diagnóstico de glaucoma, básicamente se debe saber localizar "dentro del nervio óptico", las siguientes estructuras y características: sus bordes, anillo neural, excavación fisiológica, hemorragias en el nervio y vasos en bayoneta.

Los signos de sospecha de glaucoma son el 1 aumento de excavación físiológica mayor a 0.5 en su eje vertical, a expensas de la disminución del anillo neural, los vasos emergentes en bayoneta y derivar al paciente al especialista, y si identifica una hemorragia del nervio óptico derivarlo con carácter de urgencia ${ }^{(10)}$.

\section{Obstrucciones Vasculares de la Retina}

Las obstrucciones de vena y arteria centrales de la retina producen abruptamente disminución o pérdida de la visión del ojo afectado.

\section{Obstrucción de Arteria Central de Retina (OACR)}

La arteria central de la retina es la encargada de nutrir la capa interna de la retina, por ende, una obstrucción de la misma produciría una isquemia generalizada de la retina con una marcada disminución de la visión. Es una patología poco frecuente con una incidencia de 1-10 casos/100 000 personas $^{(11)}$, mayor frecuencia de presentación en hombres y por lo general en personas mayores de 60 años. La etología con mayor asociación es de naturaleza trombótica en personas mayores con ateroesclerosis de carótidas; en personas jóvenes en cambio podría asociarse a procesos cardioembólicos ${ }^{(12)}$. Otras patologías inflamatorias vasculares como Arteritis de células gigantes y enfermedades hematológicas que producen estados de hiperviscosidad pueden asociarse a esta patología.

La historia clínica de la enfermedad es la pérdida brusca de la visión, usualmente unilateral, en ausencia de dolor. Al examen la pérdida visual suele ser severa en rango de 20/800 o peor. Se podría evidenciar defecto pupilar aferente y en la evaluación de fondo de ojo una retina pálida por la isquemia con una mancha roja cereza a nivel macular. No existen ensayos clínicos que determinen un tratamiento efectivo. El realizar masaje ocular, reducir la presión intraocular buscaría movilizar la obstrucción y recuperar la perfusión, aunque los resultados son poco exitosos ${ }^{(3)}$. Por otro lado, la terapia antitrombótica intraarterial podría traer efectos secundarios graves como hemorragias intracerebrales por lo cual no es una indicación de rutina.

Es importante la consejería al paciente y resaltar que un paciente con OACR está en riesgo de desarrollar eventos cardiovasculares y cerebrovasculares en los siguientes años, por lo que debe controlar los factores de riesgo ${ }^{(14)}$.

\section{Obstrucción de Vena Central de Retina (OVCR)}

La obstrucción de vena de la retina es la segunda causa de pérdida visual de origen vascular después de la retinopatía diabética.

La OVCR ocurre a nivel de la lámina cribosa y esta asociaba a la formación primaria de un trombo. Los pacientes acuden por visión borrosa, con ausencia de dolor, que aparece de forma súbita. La pérdida de la visión es variable, en The Central Retinal Vein Oclusion Study ${ }^{(14)}$, se realizó el seguimiento a 725 pacientes con OVCR por un período de tres años; resultados que encontraron que aquellos pacientes donde la obstrucción producía isquemia, la agudeza visual inicial era mala (peor a 20/200) en comparación de los no isquémico (agudeza visual 20/40 a 20/200). En la evaluación del paciente no se observan inicialmente alteraciones en el segmento anterior y la presión intraocular esta conservada. La evaluación de fondo de ojo se observa hemorragias en cuatro cuadrantes, edema, vasos venosos tortuosos, dilatados y podrían observarse manchas algodonosas. La angiografía con fluoresceína debe realizarse para confirmar el diagnóstico y determinar el grado de isquemia que produce la obstrucción.

El tratamiento va dirigido a las complicaciones; los pacientes que desarrollen edema macular la recomendación es la aplicación de medicamentos antiangiogénicos como primera línea; y en aquellos que desarrollen neovascularización se debe realizar fotocoagulación panretinal. 

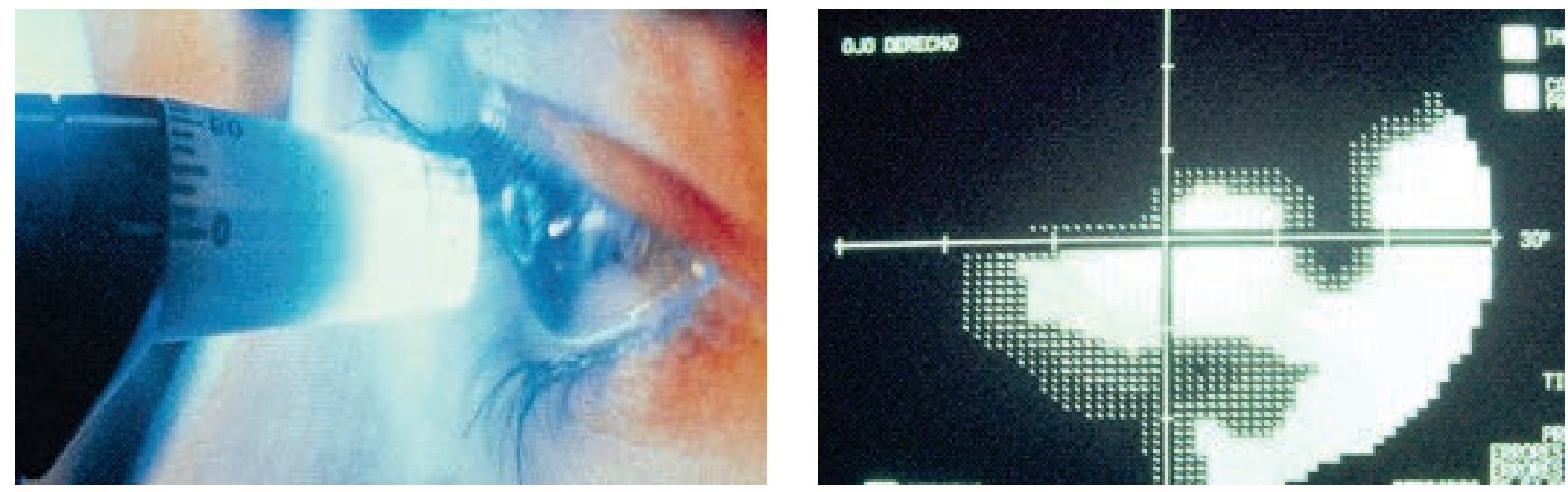

Tonometría de aplanación y campimetería computarizada en el GPAA.
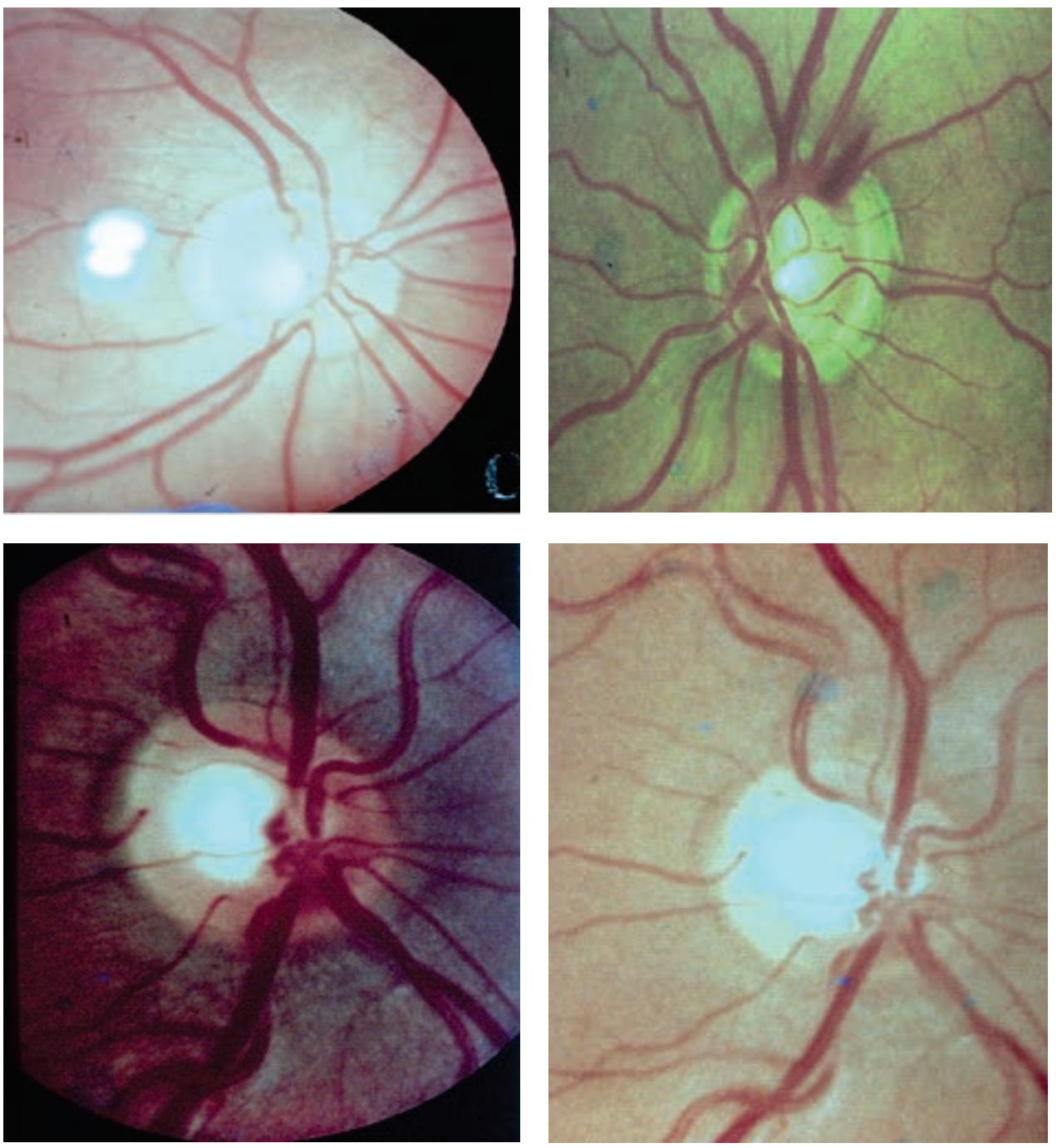

Evolución de la papila en el GPAA sin tratamiento hasta la atrofia óptica y ceguera.

\section{Neuritis Óptica}

Es la inflamación primaria del nervio óptico de diferente etiología (desmielinizante, infecciosa o idiopática).
Según la localización de la inflamación puede ser divididas en papilitis cuando se afecta solo la cabeza del nervio óptico, neurorretinitis cuando se asocian exudados retinianos en el área macular y neuritis retrobulbar cuando el fondo de ojo es normal 
y la afectación es en la porción posterior del nervio. La incidencia anual es 3-5 casos/1000000 personas años en el rango de edad entre los 20-50 años y siendo más afectadas las mujeres como se demostró en el estudio The Optic Neuritis Treatment Trial (ONTT) ${ }^{(15)}$.

La presentación clásica es la disminución súbita de visión, desde una discreta reducción hasta la no percepción de luz, alteración de la visión de colores y dolor a la motilidad ocular en $92 \%$ de los casos. Al examen se observa defecto pupilar aferente relativo, el fondo de ojo lo más frecuente es encontrar edema de papila hasta en $40 \%$ de los casos. En la prueba de campo visual se observan defectos focales como escotomas centrales o cecocentrales y defectos difusos como depresión generalizada ${ }^{(16)}$. Es importante solicitar neuroimágenes como resonancia magnética sobre todo en las presentaciones atípicas o para descartar la presencia de placas desmielinizantes que se asocian a Esclerosis múltiple. En los potenciales visuales evocados se observa un aumento de latencia en la mayoría de casos, siendo útil en casos donde la agudeza visual y el campo visual están preservados.
Referencias bibliográficas

1. Guías del Consejo Internacional de Oftalmología (ICO) para el Glaucoma. http:/www.icoph.org/downloads/ ICOGlaucoma Guidelines-Spanish.pdf

2. Salud Ocular Universal: Un plan de accion mundial para 20142019 (OMS)https://www.who.int/blindness/ AP2014 19 Spanish.pdf

3. TH Osaki TH, N Kasahara N, M D Paolera M, R Cohen R, Nishiwaki-Dantas MC. Presentation of glaucoma in an urban tertiary care hospital in South America: legal blindness and prevalence Int Ophthalmol (2010)30:361-366. doi: 10.1007/s 10792-010-935-2

4. Lopes JF, Hubatsch DA, P Amaris P. Effect of benzalkonium chloride-free travoprost on intraocular pressure and ocular surface symptoms in patients with glaucoma previously on latanoprost: an open-label study. Lopes et al. BMC Ophthalmology (2015)15:166. doi: 10.1186/s12886-015-01517

5. Quigley HA, Broman AT. The number of people with glaucoma worldwide in 2010 and 2020. Br J Ophthalmol 2006;90:262267. doi: 10.1136/bjo.2005.081224

6. Varma R, et all on behalf of the LALES Group. Four-year incidence of Open-angle Glaucoma and Ocular Hypertension: The Los Angeles Latino Eye Study. Am J Ophthalmol. 2012;154(2):315-325.e1.doi:10.1016/j.ajo. 2012.02.014

7. Furtado JM, Lansingh VC, Carter MJ, Milanese MF, Peña BN, Ghersi HA, Bote PL, Nano ME, Silva JC. Causes of Blindness and Visual Impairment in Latin America. Surv Ophthalmol 2012;57:149-177. doi:10.1016/j.survophthal.
2011.07.002

8. Melgarejo JD, et al. Glaucomatous Optic Neuropathy Associated with Nocturnal Dip in Blood Pressure. Ophthalmology 2017;-:1e8.doi.org/10.1016/j.ophtha. 2017.11.029

9.Kim E, Varma R. Glaucoma in Latinos/Hispanics. Current Opinion in Ophthalmology 2010;21:100-105. doi:10.1097/ ICU. 0b013e3283360b1e

10.Consensos del Grupo Mexicano de Investigación en Glaucoma y Colegio Mexicano de Glaucoma: Dr. Jesús Jiménez-Román. 2016.

11.Leavitt JA, Larson TA, Hodge DO, Gullerud RE. The incidence of central retinal artery occlusion in Olmsted County, Minnesota. Am J Ophthalmol 2011;152:820.

12. Yuzurihara $\mathbf{D}$, Iijima $\mathbf{H}$. Visual outcome in central retinal and branch retinal artery occlusion. Jpn J Ophthalmol 2004;48:49.

13. Nielsen NV. Treatment of acute occlusion of the retinal arteries. Acta Ophthalmol(Copenh) 1979;57:1078.

14. Natural history and clinical management of central retinal vein occlusion. The Central Vein Occlusion Study Group. Arch Ophthalmol 1997; 115:486.

15. Beck RW, Cleary PA, Backlund JC. The course of visual recovery after optic neuritis. Experience of the Optic Neuritis Treatment Trial. Ophthalmology. 1994;101(11):1771-1778.

16. Cantore WA. Optic neuritis. Pa Med. 1996;99(Suppl):96-8.

17.Fang JP, Lin RH, Donahue SP. Recovery of visual fi eld function in the optic neuritis treatment trial. Am J Ophthalmol. 1999;128(5):566-72. 